\title{
Proposal of obstacle detection and shape recognition method for transfer robot using line laser and camera
}

\author{
Miki Suetsugu, Shiyuan Yang, Seiichi Serikawa \\ Department of Electrical and Electronic Engineering, Kyushu Institute of Technology, Kitakyushu, Japan
}

*Corresponding Author: p108060m@mail.kyutech.jp

\begin{abstract}
In recent years, the increase in mail-order users tends to increase the demand for robot unmanned transport vehicles that transport packages in warehouses and factories. However, the unmanned transport vehicle currently in use runs on magnetic tape and stops when there is a load on the runway, so transport efficiency is poor. So there is a need to detect and avoid obstacles. Currently, ultrasonic sensors, PSD sensors, cameras and 2-D LiDAR are used as obstacle detection sensors. These sensors have several disadvantages. Ultrasonic sensors and PSD sensors can only measure at a point (one dimension) and are susceptible to illumination and noise. In the case of a camera, it is necessary to update the background image, which is time-consuming and costly. Although 2-D LiDAR can be measured in a wide range, it requires a drive system equipped with a rotating mirror etc. and has problems with cost and durability. In order to solve the above problems, in this research, an obstacle is detected using a line laser and a camera, and the distance to the obstacle and the shape are recognized to avoid the obstacle and transport it efficiently. We propose an obstacle recognition method. In this method, not only obstacle detection but also shape recognition can be carried out, and even if there is an obstacle on the runway, it can be flexibly avoided and efficiently transported.
\end{abstract}

Keywords: AGV, Robot, Sensor, Image processing, Camera,

Laser, Detection, Measurement.

\section{Introduction}

In recent years, the number of users of mail order is increasing worldwide. In order to support this, it is required to streamline the work of managing and transporting a large amount of luggage in a warehouse or factory. The routes of many unmanned transport vehicles currently used are determined in advance. If there is an obstacle on the route, the AGV will stop. In addition, there is a possibility of collision, which is dangerous. The technology of obstacle detection is also important for safety. In warehouses and factories, the environment in which humans and robots work together is common and needs to coexist well. Humans move irregularly. In warehouses and factories, there are various shapes other than rectangular parallelepipeds such as cardboard. Robots cannot move in anticipation of irregular movements. Moreover, it is necessary to recognize various shapes other than a box. In order to avoid these obstacles, it is necessary to detect obstacles widely and to detect them instantly.

Ultrasonic sensors, PSD sensors, cameras, and 2-D LiDAR are used as obstacle detection systems currently in use. However, these require some drawbacks. Ultrasonic sensors can be measured only in one dimension, and a large number of sensors are required to measure widely. Also, the influence of noise is large and errors are likely to occur. PSD sensors are subject to errors due to light reflected from the floor. In addition, this can also be measured in one dimension as well, and a large number is required to measure in a wide range. In the method of detecting by image processing using a camera, it is necessary to capture a background image, which is very time-consuming. In addition, information 2-D LiDAR, which is obtained at one time, requires a drive system equipped with a rotating mirror for detecting a laser. This is costly and has a drawback that it is expensive due to its complicated configuration and difficult to repair.

In order to solve the above problems, this research proposes a method to detect various obstacles using a line laser and a camera. In this way, obstacles can be widely detected. Moreover, since there is no restriction in the form, 
it can correspond to any form. The calculation is also simple and can be detected instantaneously.

\section{Obstacle recognition method of this system}

The outline of the system configuration is shown in Fig1. Fig. 1 shows a conical obstacle placed in front of the robot. As shown in Fig 1, the system mounts a green line laser on the robot and illuminates the floor. We shoot the situation with a camera.

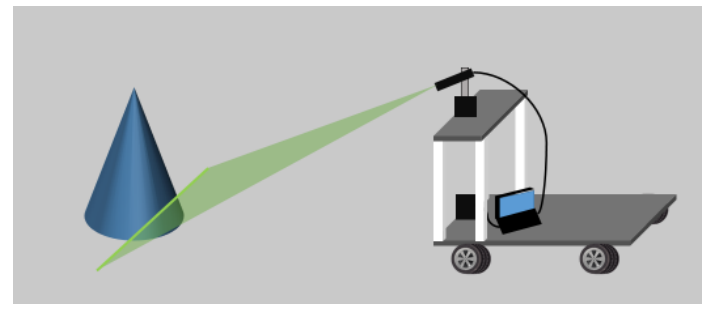

Fig. 1 Outline of this research system configuration

An example of the obtained image is shown in Fig 2. Extract the laser line from the image taken by the camera.

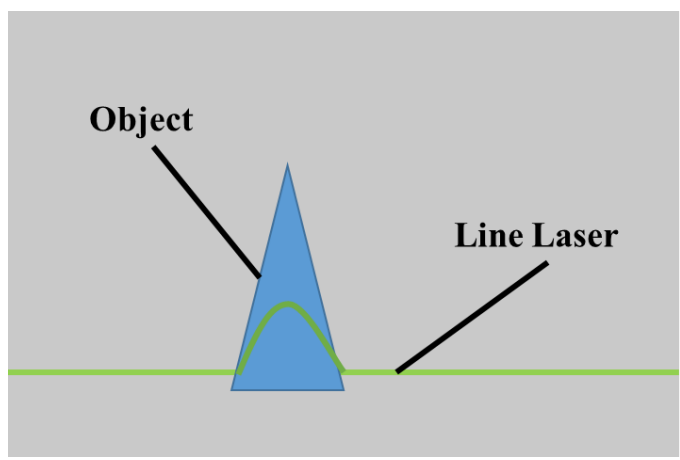

Fig. 2 Line laser irradiation

\subsection{Laser line extraction processing}

The green line laser shown in Fig.2 is extracted by image processing as follows. As preprocessing, the image is subjected to HSV conversion and mask processing. By doing so, the green line laser can be highlighted and extracted. However, since there is a possibility of extracting green other than the line laser, the area other than the line laser is erased in advance. Therefore, quick and accurate extraction is possible.

As shown in Fig. 2, when there is an obstacle in front of the robot, the straight laser line is broken. Therefore, when the straight line is broken, it can be determined that an obstacle exists in front of the robot. Before calculation, determine the position and angle of the line laser in advance.

Fig. 3(a) shows an image when there is no obstacle. Fig.
3 (b) shows an image in the case where there is a cylindrical obstacle, Fig. 3 (c) shows that in the case of a prismatic obstacle, and Fig. 3 (d) shows that in the case where there is a rectangular obstacle.

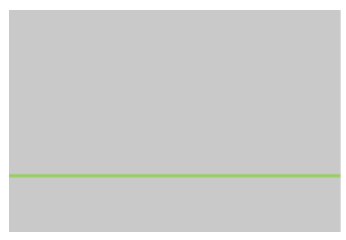

(a) No obstacle

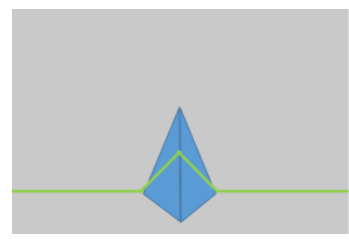

(c) Pyramid

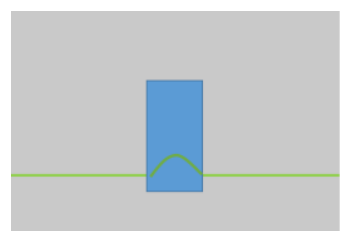

(b) Cylinder

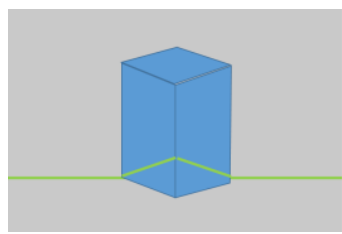

(d) Rectangular
Fig. 3 Image of line laser irradiation

As shown in Fig. 4, in order to make an accurate determination, the width is cut to the same size as the obstacle robot. The line laser also cuts the lower part where the floor is not irradiated. In this experiment, the pyramid used a square with a bottom of $13.5 \mathrm{~cm}$ and a height of 24 $\mathrm{cm}$. The rectangular parallelepiped is a rectangle $22 \mathrm{~cm}$ long and $23 \mathrm{~cm}$ wide at the bottom and $31 \mathrm{~cm}$ high. The cone is a circle with a diameter of $22 \mathrm{~cm}$ at the bottom and a height of $70 \mathrm{~cm}$. The bottom of the cylinder is $11 \mathrm{~cm}$ in diameter and $15 \mathrm{~cm}$ in height.
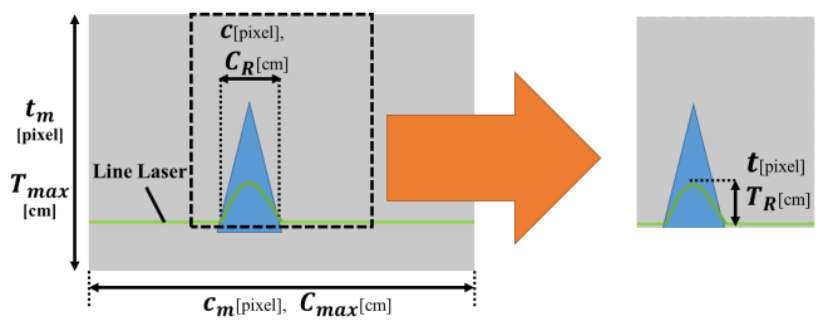

Fig. 4 Preprocessed image

\subsection{Calculation principle of the distance to the obstacle}

In this section, the distance to the obstacle is determined using the line laser extracted in Section 2.1.

As shown in Fig. 5, the distance to the obstacle is defined as D $[\mathrm{cm}]$. Here, the distance between the irradiation target of the line laser and the robot is defined as $\mathrm{L}[\mathrm{cm}]$, and the height at which the line laser is installed is defined as $\mathrm{H}[\mathrm{cm}]$.

As shown in Fig. 5, an obstacle having a slope causes a difference between the position where the line laser is 
irradiated and the distance D. It is necessary to correct this.

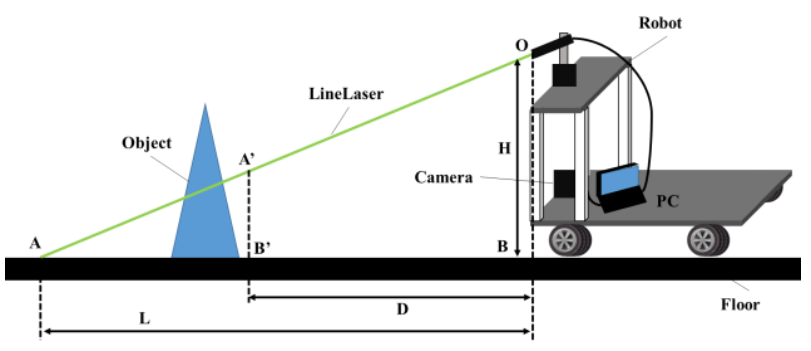

Fig5. Variable definition

\subsubsection{Calculation of height for finding distance}

Find the height $\mathrm{t}$ [pixel] of the image. First, the part under the line laser and the width under which the photographed image is irradiated to the floor are cut to the width of the robot. Extract the line laser (green) from the cut image. The height $\mathrm{T}$ is obtained from the extracted image. Then, the imaging range is determined from the irradiation destination $\mathrm{L}[\mathrm{cm}]$ of the line laser set in advance, and the imaging range and the height $T_{R}[\mathrm{~cm}]$ have a relationship of ratio. The imaging range is defined as $T_{\max }[\mathrm{cm}]$ and the vertical size $t_{m}$ [pixel] of the image size. Therefore, formula (1) is obtained.

$$
T_{R}=\frac{T_{\max } \times t}{t_{m}}
$$

\subsubsection{Distance D calculation formula}

The distance $\mathrm{D}$ is obtained from the similarity relation between two triangles. As shown in Fig. 5, there are $\triangle \mathrm{OAB}$ and $\triangle \mathrm{OA}^{\prime} \mathrm{B}$ '. The following equation (2) can be obtained using $T_{R}$ determined by equation (1).

$$
\mathrm{D}=\frac{H \cdot L}{T_{R}+H}
$$

\subsection{Obstacle width calculation principle}

As shown in Fig. 4, the width of the obstacle on the image is defined as $\mathrm{c}$ [pixel] and the maximum width $c_{m}$ [pixel]. The actual obstacle width $C_{R}[\mathrm{~cm}]$ and the imaging maximum range are $C_{\max }[\mathrm{cm}]$. The actual width and the width on the image can be expressed as a ratio. Therefore, Formula (3) can be obtained.

$$
C_{R}=\frac{C_{\max } \cdot c}{c_{m}}
$$

\section{Experiment and consideration}

\subsection{Outline of experimental system}

The outline of the system proposed in this research will be described using Fig.5. These are composed of a line laser polarized in a line shape using a semiconductor laser as a rod lens, a camera, and a personal computer. As shown in Fig. 5, the camera is placed at a low position of the mobile robot, and the line laser is placed at a high position. Shoot the line laser on the floor with a camera and input it to a PC.

In this research, the camera is EOS M10 made by Canon and the semiconductor laser is the MLXL series of Kikko Giken at a wavelength of 520 [mm].

\subsection{Obstacle height detection}

In this experiment, the distance $\mathrm{L}$ between the irradiation target of the line laser and the robot was set to 1.5 m. As obstacles, pyramids, prisms, cones, cylinders and four types of obstacles were used. Moreover, it is a material plastic and paper of an obstacle, and it is not a mirror surface or glass but a material which does not transmit light. The distance $\mathrm{D}$ between the obstacle and the robot was changed by $10 \mathrm{~cm}$ at a time. The height is measured by changing the distance $\mathrm{X}$ between the obstacle and the robot.

Table 1 summarizes the measurement results of obstacle height.

Table1 : Measurement result of height $T_{R}$

\begin{tabular}{|c|c|c|c|}
\hline \multirow{3}{*}{ Obstacle } & $\begin{array}{c}\text { Theoretical } \\
\text { value[cm] }\end{array}$ & $\begin{array}{c}\text { Measured } \\
\text { value[cm] }\end{array}$ & Error [cm] \\
\hline \multirow{3}{*}{ Pyramid } & 19.5 & 15.97 & 3.53 \\
\cline { 2 - 4 } & 13 & 9.93 & 3.07 \\
\cline { 2 - 4 } & 6.5 & 4.48 & 2.02 \\
\hline \multirow{3}{*}{ Cone } & 19.5 & 20.65 & 1.15 \\
\cline { 2 - 4 } & 13 & 12.66 & 0.34 \\
\cline { 2 - 4 } & 6.5 & 5.65 & 0.85 \\
\cline { 2 - 4 } & 19.5 & 22.97 & 3.47 \\
\hline \multirow{3}{*}{ Cylinder } & 13 & 13.26 & 0.26 \\
\cline { 2 - 4 } & 19.5 & 6.68 & 0.18 \\
\cline { 2 - 4 } & 13 & 20.84 & 1.34 \\
\hline \multirow{3}{*}{ Rectangular } & 6.5 & 5.45 & 1.05 \\
\hline
\end{tabular}


From Table 1, the error of the height of obstacles is 1.4 [cm] on average at maximum $3.53[\mathrm{~cm}]$. The higher the height, the closer the obstacle is to the robot. The higher the difference is the difference in perspective. When the theoretical value $=13,6.5[\mathrm{~cm}]$, the average error is about 1 $\mathrm{cm}$ and it can be said that the accuracy is very good. It was also found that the height can be detected regardless of the shape of the obstacle.

\subsection{Distance detection between obstacle and robot}

The distance $\mathrm{D}$ between the obstacle and the robot is calculated using the result of 3.2. The distance $\mathrm{D}$ is measured while changing by $10 \mathrm{~cm}$. The measurement results are summarized in Table 2.

Table2 : Measurement result of distance D

\begin{tabular}{|c|c|c|c|}
\hline \multirow{3}{*}{ Obstacle } & $\begin{array}{c}\text { Theoretical } \\
\text { value[cm] }\end{array}$ & $\begin{array}{c}\text { Theoretical } \\
\text { value[cm] }\end{array}$ & Error [cm] \\
\hline \multirow{4}{*}{ Pyramid } & $120(+7)$ & 125.43 & 1.57 \\
\cline { 2 - 4 } & $130(+5)$ & 134.72 & 0.28 \\
\cline { 2 - 4 } & $140(+3)$ & 143.11 & 0.11 \\
\hline \multirow{3}{*}{ Rectangular } & 120 & 118.23 & 1.77 \\
\cline { 2 - 4 } & 130 & 130.52 & 0.52 \\
\cline { 2 - 4 } & 140 & 141.31 & 1.31 \\
\hline \multirow{3}{*}{ Cone } & $120(+3)$ & 120.65 & 2.35 \\
\cline { 2 - 4 } & $130(+2)$ & 131.60 & 0.40 \\
\cline { 2 - 4 } & $140(+1)$ & 140.72 & 0.28 \\
\cline { 2 - 4 } & 120 & 117.94 & 2.06 \\
\cline { 2 - 4 } & 130 & 130.52 & 0.52 \\
\hline \multirow{3}{*}{} & 140 & 141.61 & 1.61 \\
\hline
\end{tabular}

From Table 2, the error between the obstacle and the robot is up to $2.35[\mathrm{~cm}]$ and the average is 1.07 [cm]. The pyramid and cone cause a difference in the distance D between the point irradiated by the line laser and the robot due to the inclination. The value to correct this is shown in parentheses. Since the error is a ratio of $1 \%$ with respect to the distance $\mathrm{L}=150 \mathrm{~cm}$, it can be said that the accuracy is sufficiently avoidable.

\subsection{Obstacle width detection}

If the line laser irradiated to the floor is not straight but discontinuous, it is determined that there is an obstacle in front of the robot. In this experiment, it calculates using the result of 3.2 and 3.3. The results of calculating the width $C_{R}$ of obstacles are shown in Table 3.

From Table 3, the error of the width of the obstacle is up to $1.92[\mathrm{~cm}]$ and the average is 0.67 [cm]. It can be seen that there is no difference in measurement regardless of the shape of the obstacle.

Table3 : Calculation result of width $C_{R}$ of obstacle

\begin{tabular}{|c|c|c|c|c|}
\hline Obstacle & $\begin{array}{c}\mathrm{D} \\
{[\mathrm{cm}]}\end{array}$ & $\begin{array}{c}\text { Theoretical } \\
\text { value[cm] }\end{array}$ & $\begin{array}{c}\text { Measured } \\
\text { value[cm] }\end{array}$ & $\begin{array}{c}\text { Error } \\
{[\mathrm{cm}]}\end{array}$ \\
\hline \multirow{3}{*}{ Pyramid } & 120 & 12 & 11.62 & 0.38 \\
\cline { 2 - 5 } & 130 & 15 & 15.89 & 0.89 \\
\cline { 2 - 5 } & 140 & 18 & 17.95 & 0.05 \\
\hline \multirow{3}{*}{ Rectangular } & 120 & 35 & 36.92 & 1.92 \\
\cline { 2 - 5 } & 130 & 35 & 34.27 & 0.73 \\
\cline { 2 - 5 } & 140 & 19 & 18.70 & 0.30 \\
\hline \multirow{3}{*}{ Cylinder } & 120 & 25 & 25.51 & 0.51 \\
\cline { 2 - 5 } & 130 & 30 & 29.58 & 0.42 \\
\cline { 2 - 5 } & 140 & 40 & 41.64 & 1.64 \\
\cline { 2 - 5 } & 120 & 11 & 11.35 & 0.35 \\
\cline { 2 - 5 } & 130 & 11 & 11.55 & 0.55 \\
\hline \multirow{3}{*}{} & 140 & 11 & 11.43 & 0.43 \\
\hline
\end{tabular}

\section{Conclusions}

Calculation of height $T_{R}$, calculation of distance D, and average value of calculation of width $C_{R}$ of obstacles are summarized in graphs 6 to 8 for each shape of obstacle.

There is no difference between the obstacles in the error that occurs from Fig. 6-8. The method I propose can measure widely and can measure accurately by simple processing.

The method I proposed solves the problems of the conventional method. Processing is simpler than camera processing alone. It is not susceptible to indoor lighting and light from the outside. It is not easily affected by noise. Just set the distance between the laser and the robot. Spare work can be reduced.

Furthermore, it can be detected regardless of the shape of the obstacle. The method I propose can only detect at the part where the line laser is irradiated. An obstacle with a large inclination angle cannot be detected until the line laser is irradiated. This point is a future subject.

As future issues, it is necessary to detect the depth of obstacles and to detect obstacles that are not stationary. 


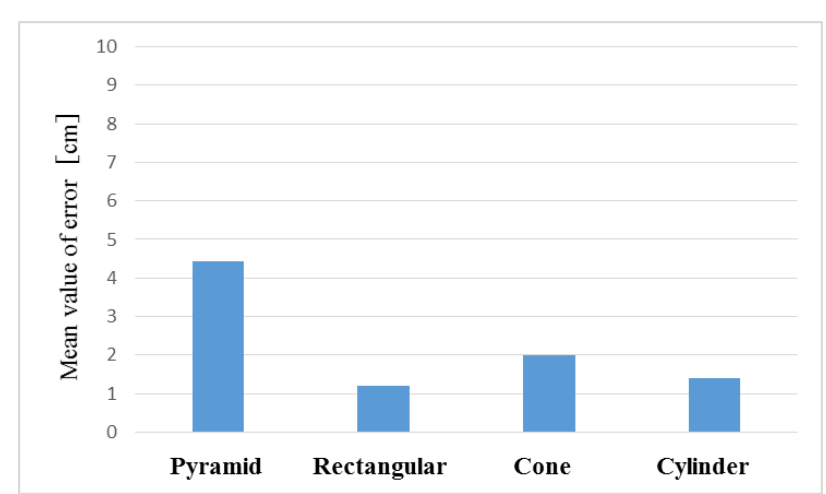

Fig6. Average error of obstacle height $T_{R}$

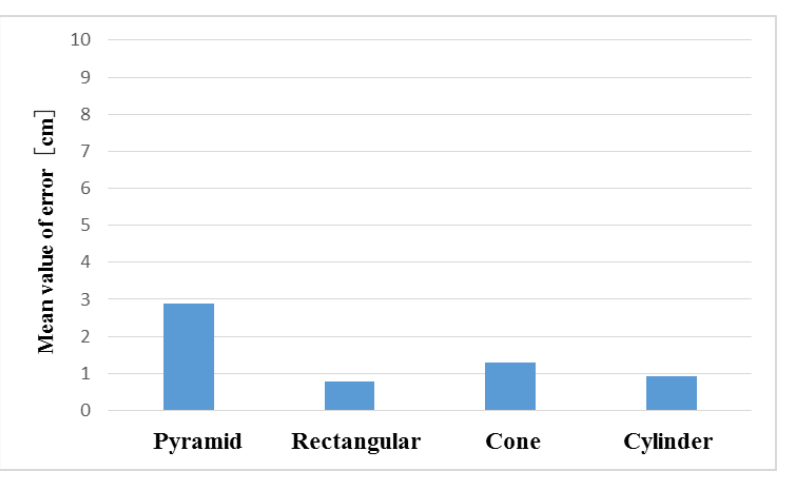

Fig7.Average error for each obstacle of distance D

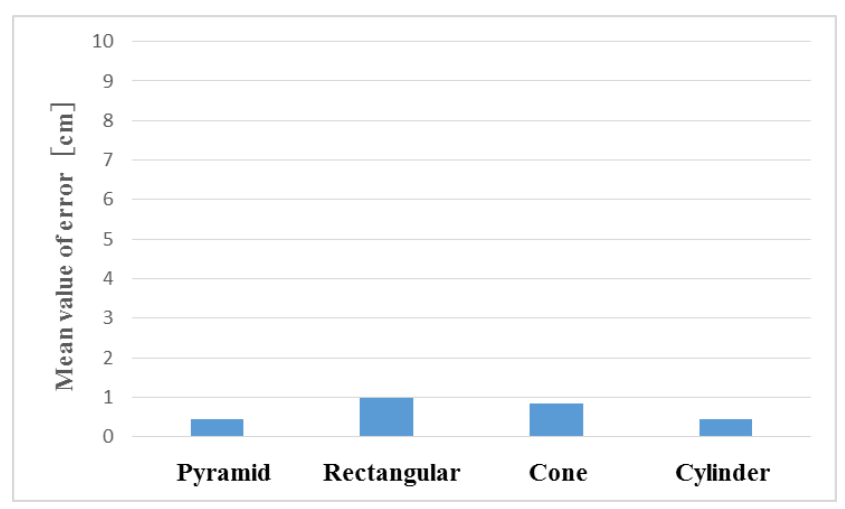

Fig8.Average error of width $C_{R}$ for each obstacle
(3) Joydeep Biswas and Manuela Veloso: "Depth camera based indoor mobile robot localization and navigation", IEEE, pp.1967-1702, 2012

(4) Florin Oniga, Sergiu Nedevschi: "Processing Dense Stereo Data Using Elevation Maps: Road Surface, Traffic Isle, and Obstacle Detection", IEEE, 59, pp.1172-1182, 2009

(5) Nils Gageik, Paul Benz and Sergio Montenegro: "Obstacle Detection and Collision Avoidance for a UAV With Complementary Low-Cost Sensors", IEEE, Volume3, pp.599-609, 2015Name : "English Title", Journal, Volume, Number, Pages, Publication Year

(6) Oe, Motoko, Tomokazu Sato, Naokazu Yokoya: "Estimating camera position and posture by using feature landmark database.", Scandinavian Conference on Image Analysis. Springer Berlin Heidelberg, pp. 171181,2005

(7) Kosei Matsumoto, Toshio Moriya and Kazuo Yano: "Obstacle avoidance system for a user guided mobile robot”, United States Patent, pp.245-264, 2011

(8) Han Wang, Zhuo Wei, Sisong Wang, Chek Seng Ow, Kah Tong Ho and Benjamin Feng: "A vision-based obstacle detection system for Unmanned Surface Vehicle", IEEE, 5, pp.364-369, 2011

\section{References}

(1) Jeong-Gi Lee, D.Kim, Jin-Young Suk: "Development of a 3D Position Determination Device using PSD sensor", IEEE, pp.1733-1736, 2010

(2) Hidenori Ishihara, Toshio Fukuda, Kazuhiro Kosuge and F.Arai : "An approach to autonomous microrobot micro line trace robot with reflex algorithm", IEEE /ASME Transactions, Vol. 1, No. 1, pp.68-79, 1996 